\title{
Hygrothermal postbuckling analysis of smart multiscale piezoelectric composite shells
}

\author{
Mahsa karimiasl $^{1}$, Farzad Ebrahimi $^{1, a}$, Vinyas Mahesh ${ }^{2,3}$ \\ ${ }^{1}$ Department of Mechanical Engineering, Faculty of Engineering, Imam Khomeini International University, \\ Qazvin, Iran \\ 2 Department of Aerospace Engineering, Indian Institute of Science, Bangalore 560012, India \\ ${ }^{3}$ Department of Mechanical Engineering, Nitte Meenakshi Institute of Technology, Bangalore 560064, India
}

Received: 5 March 2019 / Accepted: 19 November 2019 / Published online: 10 February 2020

(C) Società Italiana di Fisica (SIF) and Springer-Verlag GmbH Germany, part of Springer Nature 2020

\begin{abstract}
In this article, the hygrothermal postbuckling behaviour of multiscale doubly curved piezoelectric shells (MDCPS) has been evaluated through multiple scales perturbation method. The multiscale composite constituting of polymer/carbon nanotube/fiber and polymer/graphene platelet/fiber (PGF) in accordance with Halpin-Tsai model is considered for evaluation. The kinematics of the MDCPS is assumed to follow third order shear deformation theory (TSDT) and the non-linearity in the strain-displacement relation is adjudged through Von Karman relationship. Further, with the aid of Hamilton's principle the governing equations of motion are derived. The present work studies the load-deflection curves for various distribution patterns such as $U$ (uniform), $X, A$ and $O$. In addition, the influence of various prominent parameters like applied voltage, magnetic potential, aspect and curvature ratio on the postbuckling characteristics of MDCPS is studied thoroughly.
\end{abstract}

\section{Introduction}

Recently, numerous engineering applications have witnessed the tremendous usage of smart materials due to its various beneficial properties including adaptability, quick response time, sensing and actuating capability etc. Further, when these smart materials are incorporated in the engineering structures in the form of fibers, its efficiency significantly improves due to reduced defects. Among the many smart materials, piezoelectric (PE) are the potential candidate for sensor and actuator due to its unique energy conversion capability. Therefore, incorporating PE materials as a fiber in the composite materials has set a new trend in smart composite materials. In this direction, many significant literatures have been reported to understand the prominence of smart composite materials. Odegard et al. [1] and Gao and $\mathrm{Li}$ [2] attempted to evaluate the constitutive properties of various nanotube materials through multiscale models derived via micromechanics and atomic simulations. Chen et al. [3] studied the mechanical behaviour of single polymer-CNT incorporating finite element (FE) analysis.

In contrast to beams and plates, a unique structural response is exhibited by shells due to its geometrical characteristics. Therefore, many pioneers have attempted to investigate

\footnotetext{
a e-mail: febrahimy@eng.ikiu.ac.ir
} 
different structural responses of composite shells. Naidu et al. [4] incorporated first-order shear deformation theory (FSDT) and developed a FE formulation to investigate the influence of hygrothermal environment on the non-linear vibration response of composite shells. Through higher order shear deformation theory, Amabili et al. [5] and Singh et al. [6] justified that the non-linear term plays an important role in predicting the non-linear frequencies of composite shells. Chofi and Houmat [7] assessed the natural frequency characteristics of functionally graded material (FGM) doubly curved shallow shell via FE methods and also evaluated the influence of various geometrical and material parameters on the vibration response. Alijani et al. [8] adapted Donnell's non-linear strain-displacement relation and probed the structural responses of FGM shallow shell by multiple scales analytical method. The effect of elastic foundation on the natural frequencies of FGM doubly curved shell is evaluated by Shen et al. [9]. They incorporated von-Karman non-linear strain-displacement in work shear deformation theory to find the solution to the frequency problem. Among the various effective computational methods, Homotopy analysis method is considered to be prominent [10]. Incorporating homotopy perturbation method (HPM) method, Yazdi et al. [11] investigated the non-linear vibration response of doubly curved cross-ply shell. Panda et al. [12] proposed a FE formulation and assessed the postbuckling characteristics of shape memory alloy (SMA) reinforced composite shell. The effect of external thermal loading on the non-linear stability response of smart composite shell with piezoelectric layers was probed by Pradyumna et al. [13]. Their research claimed that the location of piezoelectric layers has a significant influence. They made use of FSDT to accomplish this objective. Analogously, the effect of cracks on the non-linear bending characteristics of CNTRC shells was investigated by Fan and Wang [14]. Heydari et al. [15] incorporated differential quadrature method to understand the non-linear bending characteristics of functionally graded/CNT plates resting on elastic foundation and acted upon by uniform pressure. Rafiee et al. [16] and Razavi et al. [17] carried out the dynamic analysis of FGM shells and composite hybrid plates subjected to electro-thermo-mechanical, respectively. The study revealed that a significant effect of piezoelectric thickness applied voltage and magnetic potential prevailed.

The structures exposed to thermal environment behave uniquely when compared to mechanical loading alone. Therefore, evaluating the influence of thermal loads of the mechanical responses is of prime importance. Using perturbation method in association with HSDT, Shen et al. [18] studied the effect of thermal environment on the non-linear dynamic characteristics of functionally graded graphene-reinforcement plates resting on elastic foundation. El-Haina et al. [19] devoted his research to analyzing the stability behaviour of thermal loaded thick FG sandwich plate using sinusoidal shear deformation theory along with the stress function. A similar analysis was proposed by Menasria et al. [20] through FSDT. The bending response study of FG plates with temperature-dependent material properties was attempted by Attia et al. [21] under the framework of HSDT. Chikh et al. [22] presented a modified HSDT with a new displacement field to assess the stability of cross-ply laminated composite plates under thermal environment. Similarly, a four-variable trigonometric plate theory was proposed by Beldjelili et al. [23], to investigate the problem pertaining to the bending response of S-FGM plates with elastic foundation, subjected to hygro-thermomechanical loading. Similarly, the thermo-bending response study of FG sandwich plate [24, 25], FG plates resting on elastic medium [26, 27] has been reported. The effect of different through-thickness varying thermal environment on the buckling behaviour of FGM plate was analyzed by Bousahla et al. [28].

On the other hand, various researches were parallely carried out with respect to nanostructures as well. Besseghier et al. [29] proposed a novel non-local refined trigonometric 
shear deformation theory and evaluated the dynamic response of FG nanoplates lying of elastic foundation. The effect of thermal environment on frequency of single-walled carbon nanotubes (SWCNTs) was assessed by Cherif et al. [30]. Khetir et al. [31] used FSDT and demonstrated the buckling nature of FG nanoplate lying on elastic foundation and subjected to different thermal loading profiles. For FG nanobeams, Mouffoki et al. [32] extended the similar evaluation considering the effect of moisture via novel simple trigonometric shear deformation theory.

The research community has witnessed the development of various novel theories to assess the mechanical behaviour of various macro and nano structures. Among them, Abdelaziz et al. [33] and Meksi et al. [34] proposed a new hyperbolic shear deformation theory and demonstrated the structural characteristics of FGM sandwich plates. Bellifa et al. [35] developed four variable refined plate theories to investigate the buckling analysis of FG plates. A novel HSDT was proposed by Zine et al. [36] to probe mechanical responses of multilayered plates and shells. The characteristic behaviour of exponentially FG sandwich plate was evaluated by Meziane et al. [37]. Also, few more literatures dealing with free vibration [38-42], static [43-48] and buckling [49] characteristics of FG, sandwich composite structures considering stretching effect have found be handy is understanding the structural behaviour for various applications.

Among the literatures on nanostructures, Bouadi et al. [50] and Mokhtar et al. [51] derived novel non-local shear deformation theories which encapsulates a new displacement field which improves the accuracy of assessing buckling properties of single-layer graphene sheet (SLGS). Similarly, Yazid et al. [52] proposed a new non-local refined plate theory and evaluated the buckling response of SLGS on elastic foundation. Bouafia et al. [53] incorporated the thickness stretching effects through a non-local quasi-3D theory and evaluated the static and dynamic response of FG nanobeams. A similar study has been reported by Zemri et al. [54], Ahouel et al. [55]. Bellifa et al. [56] demonstrated the non-linear postbuckling behavior of nanoscale beams. In their proposed formulation, unlike conventional shear deformation theories, instead of rotational displacement, the shear deformation effect is considered in the axial displacement. Incorporating small-scale effects with FSDT, Kadari et al. [57] investigated the buckling response of orthotropic nanoplates by proposing a new hyperbolic plate theory. Karami et al. [58] proposed a new non-local HSDT in association with non-local strain gradient elasticity theory to evaluate the wave dispersion in anisotropic doubly-curved nanoshells. In extension to this, Karami et al.'s [59] study performed the mechanical analysis of anisotropic nanoparticles as well. The dynamic analysis of carbon nanotube-reinforced composite (CNTRC) plates with elastic foundation was carried out by Bakhadda et al. [60]. Bounouara et al. [61] presented the free vibration analysis of functionally graded (FG) nanoscale plates resting on elastic foundation. Larbi Chaht et al. [62] considered thickness stretching effect and demonstrated the bending and buckling behaviour of FG nanobeams. Apart from the various computation techniques mentioned above, fractional calculus approach [63-73] is also proved to be handy in solving various structural problems including different boundary value problems [74-78].

Finally, it can be mentioned that this article is presented the hygrothermal buckling of multiscale doubly curved piezoelectric shell (MDCPS) with different distribution pattern such as $X, A, O, U$. The equations of motion are constructed in frame work third order shear deformation theory (TSDT) and Von-Karman type geometric non-linearity. In addition, the influences of aspect ratio, curvature ratio, moisture-temperature variation, applied voltage, magnetic potential and various pattern distributions on critical buckling are studied. The aim of this research is to develop a new smart model of sandwich composite shell. Furthermore, motion equations will be derived using Hamilton's principle and will be solved according 
to multiple scale perturbation method. Effect of important parameters on the non-linear frequency of the sandwich composite shell will be considered.

\section{Theory and formulation}

\subsection{Multiscale model for sandwich composite}

The effective constituent of the multi-layer PCF and PGF multiscale composite can be presented via Halpin-Tsai model [79] and micromechanics approaches of scheme have been expressed by Shen et al. [80]. The credibility of using this multiscale model can be justified by its efficiency of incorporating both micro/nano scale mechanics in association with the macro mechanics of the composites. The application of modeling of macroscale composites can be witnessed in various aerospace structures, smart structures, bioengineering tools etc.

The properties of the PCF and PGF shell are concentrated to be orthotropic can be presented as [80]:

$$
\begin{gathered}
E_{11}=V_{f} E_{11}^{F}+V_{\mathrm{mcn}} / \mathrm{mgpl} \\
\frac{1}{E_{22}}=\frac{1}{E_{11}^{F}}+\frac{V_{\mathrm{mcn} / \mathrm{mgpl}}}{E_{\mathrm{mcn} / \mathrm{mgpl}}}-V_{f} V_{\mathrm{mnc} / \mathrm{mgpl}}-\frac{\frac{V_{f}^{2} E_{\mathrm{mcn} / \mathrm{mgpl}}}{E_{22}^{F}}+\frac{V_{\mathrm{mcn} / \mathrm{mgpl}}^{2} E_{\mathrm{mcn} / \mathrm{mgpl}}}{E^{\mathrm{mcn} / \mathrm{mgpl}}}-2 V_{f} V_{\mathrm{mcn} / \mathrm{mgpl}}}{V_{f} E_{22}^{F}+V_{\mathrm{mcn} / \mathrm{mgpl}} E_{\mathrm{mcn} / \mathrm{mgpl}}} \\
\frac{1}{G_{12}}=\frac{V_{f}}{G_{11}^{F}}+\frac{V_{\mathrm{mcn} / \mathrm{mgpl}}}{G_{\mathrm{mcn} / \mathrm{mgpl}}} \\
\rho=V_{f} \rho_{f}+V_{\mathrm{mcn} / \mathrm{mgpl}} \rho_{\mathrm{mcn} / \mathrm{mgpl}} \\
\vartheta_{12}=V_{f} v_{f}+V_{\mathrm{mcn} / \mathrm{mgpl}} v_{\mathrm{mcn} / \mathrm{mgpl}}
\end{gathered}
$$

where $E_{11}^{F}, E_{22}^{F}$ are the Young's modulus of CNT/GPL, $G_{12}$ shear modulus and $\rho$ is mass density, $\vartheta_{12}$ Poisson's ratio of fibers, respectively, the corresponding properties of the isotropic matrixes of CNT/GPL composite presented with $E_{\mathrm{mcn} / \mathrm{mgpl}}, G_{\mathrm{mcn} / \mathrm{mgpl}}, \rho_{\mathrm{mcn} / \mathrm{mgpl}}$ and $V_{\mathrm{mcn} / \mathrm{mgpl}}$ and volume fractions of the fiber presented by $V_{f}$.

Via Halpin-Tsai model, composites tensile modulus has been expressed [81]:

$$
\begin{gathered}
E_{\mathrm{mcn} / \mathrm{mgpl}}=\frac{E_{M}}{8}\left[5\left(\frac{1+2 \beta_{\mathrm{dd}} V_{\frac{\mathrm{cn}}{\mathrm{gpl}}}}{1-\beta_{\mathrm{dd}} V_{\frac{\mathrm{cn}}{\mathrm{gpl}}}}\right)+3\left(\frac{1+2\left(\frac{l_{\mathrm{cn}}}{d_{\frac{\mathrm{cn}}{\mathrm{gpl}}}}\right) \beta_{l l} V_{\frac{\mathrm{cn}}{\mathrm{gpl}}}}{1-\beta_{l l} V_{\frac{\mathrm{cn}}{\mathrm{gpl}}}}\right),\right. \\
\beta_{l l}=\frac{\frac{E_{11}^{\mathrm{cn} / \mathrm{gpl}}}{E_{M}}-\left(\frac{d_{\mathrm{cn} / \mathrm{gpl}}}{4 t^{\mathrm{cn} / \mathrm{gpl}}}\right)}{\frac{E_{11}^{\mathrm{cn} / \mathrm{gpl}}}{E_{M}}+\left(\frac{l_{\mathrm{cn} / \mathrm{gpl}}}{2 h^{\mathrm{cn} / \mathrm{gpl}}}\right)}, \\
\beta_{\mathrm{dd}}=\frac{\frac{E_{11}^{\mathrm{cn} / \mathrm{gpl}}}{E_{M}}-\left(\frac{d_{\mathrm{cn} / \mathrm{gpl}}}{4 h^{\mathrm{cn} / \mathrm{gpl}}}\right)}{\frac{E_{11}^{\mathrm{cn} / \mathrm{gpl}}}{E_{M}}+\left(\frac{d_{\mathrm{cn} / \mathrm{gpl}}}{2 h^{\mathrm{cn} / \mathrm{gpl}}}\right)}
\end{gathered}
$$


where $E_{11}^{\mathrm{cn} / \mathrm{gpl}}$, refers to the Young's modulus, $h^{\mathrm{cn} / \mathrm{gpl}}, d_{\mathrm{cn} / \mathrm{gpl},}, l_{c n / g p l}$ presented thickness, outer diameter, length and $V_{\mathrm{cn} / \mathrm{gpl}}$ are the volumes fraction of carbon nanotubes and graphene platelet, respectively, and $V_{\mathrm{mcn} / \mathrm{gpl}}$ and $E_{\mathrm{mcn} / \mathrm{mgpl}}$ are the volumes fraction of the matrices and Young's modulus, respectively.

For the different distribution multiscale composite shell, the weight fraction of CNT and GPL changes layerwise in accordance with the according distribution pattern such as $U$, $X, A$ and $O$ are studied. CNT and GPL volume fraction of $n$th layer corresponding to each distribution pattern can be presented as [82]:

$$
\begin{gathered}
U: V_{\mathrm{cn} / \mathrm{gpl}}^{n}=V_{\mathrm{cn} / \mathrm{gpl}}, \\
X: V_{\mathrm{cn} / \mathrm{gpl}}^{n}=2 V_{\frac{\mathrm{cn}}{\mathrm{gpl}}}\left(\frac{\left|2 n-n_{t}-1\right|}{n_{t}}\right), \\
O: V_{\mathrm{cn} / \mathrm{gpl}}^{n}=2 V_{\mathrm{cn} / \mathrm{gpl}}\left(1-\frac{\left|2 n-n_{t}-1\right|}{n_{t}}\right), \\
A: V_{\mathrm{cn} / \mathrm{gpl}}^{n}=V_{\mathrm{cn} / \mathrm{gpl}}\left(\frac{|2 n-1|}{n_{t}}\right)
\end{gathered}
$$

where, the total number of layers can be expressed by $n_{t}$ and the total volumes fraction of CNT GPL can be presented by [83]:

$$
V_{\mathrm{cn} / \mathrm{gpl}}=\frac{w_{\mathrm{cn} / \mathrm{gpl}}}{w_{\mathrm{cn}}+\left(\frac{\rho_{\mathrm{cn} / \mathrm{gpl}}}{\rho_{m}}\right)-\left(\frac{\rho_{\mathrm{cn} / \mathrm{gpl}}}{\rho_{m}}\right) w_{\mathrm{cn}}}
$$

where $\rho_{c n / g p l}$ are the mass densities of the CNT and GPL and $\rho_{m}$ is epoxy resin matrix, $w_{c n / g p l}$ are the mass fraction of the CNT and GPL, respectively.

The mass densities of CNT and GPL can be presented as:

$$
\begin{gathered}
\rho_{m n c / m g p l}=V_{c n / g p l} \rho_{c n / g p l}+v_{m} \rho_{m}, \\
G_{m n c / m g p l}=\frac{E_{m n c / m g p l}}{2\left(1+v_{m c n / m g p l}\right)}, \\
V_{m c n / m g p l}=V_{m}
\end{gathered}
$$

where, $v_{m}, v_{m c n / m g p l}$ is the Poisson's ratio of the matrix, CNT, GPL and $\alpha_{11}$ and $\alpha_{22}$ is the thermal expansion coefficients in longitudinal and transverse directions [80]. So $\alpha_{11}^{f}$ is the thermal expansion coefficient of longitudinal fiber and $\alpha_{22}^{f}$ presented in transverse directions of the fiber. $\alpha_{m c n / m g p l}$ can be expressed as [84]:

$$
\begin{gathered}
\alpha_{11}=\frac{V_{f} E_{11}^{f} \alpha_{11}^{f}+V_{m c n / m g p l} E_{m c n / m g p l} \alpha_{m c n / m g p l}}{V_{f} E_{11}^{f}+V_{m c n / m g p l} E_{m c n} / m g p l}, \\
\alpha_{22}=\left(1+V_{f}\right) V_{f} \alpha_{22}^{f}+\left(1+V_{m n c / m g p l}\right) V_{m c n / m g p l} \alpha_{m c n / m g p l}-v_{12} \alpha_{11}, \\
\alpha_{m c n}=\frac{1}{2}\left\{\left(\frac{V_{c n / g p l} E_{c n / g p l} \alpha_{c n / g p l}+v_{m} E_{m} \alpha_{m}}{v_{c n / g p l} E_{c n / g p l}+v_{m} E_{m}}\right)\right\}\left(1-v_{m c n / m g p l}\right) \\
+\left(1+v_{m}\right) \alpha_{m} V_{m}+\left(1+v_{c n / m g p l}\right) \alpha_{c n / m g p l} V_{c n / m g p l}
\end{gathered}
$$


where $\alpha_{m c n} / m g p l, \beta_{m c n / m g p l}$, are the thermal expansion and moisture coefficients of the epoxy resin CNT and GPL matrix and $\alpha_{c n / g p l}$ are the thermal expansion coefficients of the CNT and GPL, respectively.

$$
\begin{gathered}
\beta_{11}=\frac{V_{f} E_{11}^{f}+V_{m c n / m g p l} E_{m c n / m g p l} \beta_{m}}{V_{f} E_{11}^{f}+V_{m c n / m g p l} E_{m c n / m g p l}}, \\
\beta_{22}=\left(1+V_{m c n / m g p l}\right) V_{m c n / m g p l} \beta_{m}-v_{12} \beta_{11}
\end{gathered}
$$

\section{Kinematic relations}

In the frame work of TSDT, the displacement fields at an arbitrary point in the composite shell can be expressed as:

$$
\begin{gathered}
u=u_{0}+z \varphi_{x}-\frac{4}{3 h^{2}} z^{3}\left(\varphi_{x}+\frac{\partial w_{0}}{\partial x}\right) \\
v=v_{0}+z \varphi_{y}-\frac{4}{3 h^{2}} z^{3}\left(\varphi_{y}+\frac{\partial w_{0}}{\partial y}\right), \\
w=w_{0}
\end{gathered}
$$

In these equations, $u_{0}, v_{0}$, and $w_{0}$ are the original displacements of the shell in the $x$, $y$ directions; the rotations of transverse normal at the mid-plane in the $x$ and $y$ axes represented by $\varphi_{x}$ and $\varphi_{y} \cdot u_{0}, v_{0}, w_{0}, \varphi_{x}, \varphi_{y}$ are functions of the five original variables which describe the shell displacements. Von Karman type geometric non-linearity, the strain components $\varepsilon_{x x}$, $\varepsilon_{y y}$ and $\gamma_{x y}$ can be shown as:

$$
\begin{aligned}
& \left\{\begin{array}{l}
\varepsilon_{x x} \\
\varepsilon_{y y} \\
\gamma_{x y}
\end{array}\right\}=\left\{\begin{array}{l}
\varepsilon_{x x}^{0} \\
\varepsilon_{y y}^{0} \\
\gamma_{x y}^{0}
\end{array}\right\}+z\left\{\begin{array}{l}
k_{x x}^{0} \\
k_{y y}^{0} \\
k_{x y}^{0}
\end{array}\right\}+z^{3}\left\{\begin{array}{l}
k_{x x}^{2} \\
k_{y y}^{2} \\
k_{x y}^{2}
\end{array}\right\} \\
& =\left\{\begin{array}{l}
\frac{\partial u_{0}}{\partial x}+\frac{w_{0}}{R_{1}}+\frac{1}{2}\left(\frac{\partial w_{0}}{\partial x}\right)^{2} \\
\frac{\partial v_{0}}{\partial y}+\frac{1}{2}\left(\frac{\partial w_{0}}{\partial y}\right)^{2}+\frac{w_{0}}{R_{2}} \\
\frac{\partial u_{0}}{\partial y}+\frac{\partial w}{\partial x} \frac{\partial w}{\partial y}+\frac{\partial v_{0}}{\partial x}
\end{array}\right\}+z\left\{\begin{array}{c}
\frac{\partial \varphi_{x}}{\partial x} \\
\frac{\partial \varphi_{y}}{\partial y} \\
\frac{\partial \varphi_{x}}{\partial y}+\frac{\partial \varphi_{y}}{\partial x}
\end{array}\right\} \\
& -\frac{4}{3 h^{2}} z^{3}\left\{\begin{array}{c}
\frac{\partial \varphi_{x}}{\partial x}+\left(\frac{\partial^{2} w_{0}}{\partial x^{2}}\right) \\
\frac{\partial \varphi_{y}}{\partial y}+\left(\frac{\partial^{2} w_{0}}{\partial y^{2}}\right) \\
\frac{\partial \varphi_{x}}{\partial y}+\frac{\partial \varphi_{y}}{\partial x}+2\left(\frac{\partial^{2} w_{0}}{\partial x \partial y}\right)
\end{array}\right\} \\
& \left\{\begin{array}{c}
\tau_{y z} \\
\tau_{x z}
\end{array}\right\}=\left\{\begin{array}{c}
\gamma_{y z}^{0} \\
\gamma_{x z}^{0}
\end{array}\right\}+z^{2}\left\{\begin{array}{l}
k_{y z}^{1} \\
k_{x z}^{1}
\end{array}\right\}=\left\{\begin{array}{l}
\varphi_{y}+\frac{\partial w_{0}}{\partial y} \\
\varphi_{x}+\frac{\partial w_{0}}{\partial x}
\end{array}\right\}-\frac{4}{h^{2}} z^{3}\left\{\begin{array}{l}
\varphi_{y}+\frac{\partial w_{0}}{\partial y} \\
\varphi_{x}+\frac{\partial w_{0}}{\partial x}
\end{array}\right\}
\end{aligned}
$$


Based on Maxwell's equation the electric and magnetic potential distributions can be obtained as:

$$
\begin{aligned}
& \Phi(x, z, t)=-\cos (\xi z) \phi(x, t)+\frac{2 z}{h} V, \\
& \psi(x, z, t)=-\cos (\xi z) \psi(x, t)+\frac{2 z}{h} \Omega
\end{aligned}
$$

where $\xi=\pi / h$. Also, $V$ and $\omega$ are the external electric voltage and magnetic potential applied.

Via Maxwell's equation, the relations between electric field $\left(E_{x}, E_{y}, E_{z}\right)$ and electric potential and also magnetic field $\left(Q_{x}, Q_{y}, Q_{z}\right)$ and magnetic potential can be expressed as:

$$
\begin{gathered}
E_{x}=-\phi_{, x}=\cos (\xi z) \frac{\partial \phi}{\partial x} \quad Q_{x}=-\psi_{, x}=\cos (\xi z) \frac{\partial \psi}{\partial x} \\
E_{y}=-\phi_{, y}=\cos (\xi z) \frac{\partial \phi}{\partial y} \quad Q_{y}=-\psi_{, y}=\cos (\xi z) \frac{\partial \psi}{\partial y} \\
E_{z}=-\phi_{, z}=\xi \sin (\xi z)-\frac{2 v}{h} \quad Q_{z}=-\psi_{, z}=\xi \sin (\xi z)-\frac{2 v}{h}
\end{gathered}
$$

The constitutive relation of the composite shell with piezoelectric layer, if the fiber angle with the geometric $x$ axis is expressed by $\theta$, the relation stress-strain can be transferred to the geometric coordinates as:

$$
\begin{aligned}
\left\{\begin{array}{l}
D_{x} \\
D_{y} \\
0 \\
D_{z} \\
0
\end{array}\right\}= & {\left[\begin{array}{lllll}
0 & 0 & 0 & 0 & e_{15} \\
0 & 0 & e_{15} & 0 & 0 \\
0 & 0 & 0 & 0 & 0 \\
e_{31} & e_{31} & 0 & 0 & 0 \\
0 & 0 & 0 & 0 & 0
\end{array}\right]\left\{\begin{array}{l}
\varepsilon_{x x}-\lambda_{3}\left(T(z)-T_{0}\right) \\
\varepsilon_{y y} \\
\gamma_{y z} \\
\gamma_{x y} \\
\gamma_{x z}
\end{array}\right\} } \\
& {\left[\begin{array}{lllll}
s_{11} & 0 & 0 & 0 & 0 \\
0 & s_{11} & 0 & 0 & 0 \\
0 & 0 & 0 & 0 & 0 \\
0 & 0 & 0 & s_{33} & 0 \\
0 & 0 & 0 & 0 & 0
\end{array}\right]\left\{\begin{array}{l}
E_{x} \\
E_{y} \\
0 \\
E_{z} \\
0
\end{array}\right\} } \\
& -\left[\begin{array}{lllll}
d_{11} & 0 & 0 & 0 & 0 \\
0 & d_{11} & 0 & 0 & 0 \\
0 & 0 & 0 & 0 & 0 \\
0 & 0 & 0 & d_{33} & 0 \\
0 & 0 & 0 & 0 & 0
\end{array}\right]\left\{\begin{array}{l}
Q_{x} \\
Q_{y} \\
0 \\
Q_{z} \\
0
\end{array}\right\}
\end{aligned}
$$




$$
\begin{aligned}
& \left\{\begin{array}{l}
D_{x} \\
D_{y} \\
0 \\
D_{z} \\
0
\end{array}\right\}=\left[\begin{array}{lllll}
0 & 0 & 0 & 0 & e_{15} \\
0 & 0 & e_{15} & 0 & 0 \\
0 & 0 & 0 & 0 & 0 \\
e_{31} & e_{31} & 0 & 0 & 0 \\
0 & 0 & 0 & 0 & 0
\end{array}\right]\left\{\begin{array}{l}
\varepsilon_{x x}-\lambda_{3}\left(T(z)-T_{0}\right) \\
\varepsilon_{y y} \\
\gamma_{y z} \\
\gamma_{x y} \\
\gamma_{x z}
\end{array}\right\} \\
& -\left[\begin{array}{lllll}
s_{11} & 0 & 0 & 0 & 0 \\
0 & s_{11} & 0 & 0 & 0 \\
0 & 0 & 0 & 0 & 0 \\
0 & 0 & 0 & s_{33} & 0 \\
0 & 0 & 0 & 0 & 0
\end{array}\right]\left\{\begin{array}{l}
E_{x} \\
E_{y} \\
0 \\
E_{z} \\
0
\end{array}\right\} \\
& -\left[\begin{array}{lllll}
d_{11} & 0 & 0 & 0 & 0 \\
0 & d_{11} & 0 & 0 & 0 \\
0 & 0 & 0 & 0 & 0 \\
0 & 0 & 0 & d_{33} & 0 \\
0 & 0 & 0 & 0 & 0
\end{array}\right]\left\{\begin{array}{l}
Q_{x} \\
Q_{y} \\
0 \\
Q_{z} \\
0
\end{array}\right\} \\
& \left\{\begin{array}{c}
B_{x} \\
B_{y} \\
0 \\
B_{z} \\
0
\end{array}\right\}=\left[\begin{array}{ccccc}
0 & 0 & 0 & 0 & q_{15} \\
0 & 0 & q_{15} & 0 & 0 \\
0 & 0 & 0 & 0 & 0 \\
q_{31} & q_{31} & 0 & 0 & 0 \\
0 & 0 & 0 & 0 & 0
\end{array}\right]\left\{\begin{array}{c}
\varepsilon_{x x}- \\
\varepsilon_{y y} \\
\gamma_{y z} \\
\gamma_{x y} \\
\gamma_{x z}
\end{array}\right\}-\left[\begin{array}{ccccc}
d_{11} & 0 & 0 & 0 & 0 \\
0 & d_{11} & 0 & 0 & 0 \\
0 & 0 & 0 & 0 & 0 \\
0 & 0 & 0 & d_{33} & 0 \\
0 & 0 & 0 & 0 & 0
\end{array}\right]\left\{\begin{array}{c}
E_{x} \\
E_{y} \\
0 \\
E_{z} \\
0
\end{array}\right\} \\
& -\left[\begin{array}{ccccc}
\chi_{11} & 0 & 0 & 0 & 0 \\
0 & \chi_{11} & 0 & 0 & 0 \\
0 & 0 & 0 & 0 & 0 \\
0 & 0 & 0 & \chi_{33} & 0 \\
0 & 0 & 0 & 0 & 0
\end{array}\right]\left\{\begin{array}{c}
Q_{x} \\
Q_{y} \\
0 \\
Q_{z} \\
0
\end{array}\right\}
\end{aligned}
$$

$\sigma_{i j}, D_{i j}, B_{i j}, \varepsilon_{i j}, E_{n}$ and $Q_{n}$ expressed the magnitude of stress, electric displacement, magnetic induction, non-linear strain, electric field and magnetic field. Also, $s_{i j}$ and $\chi_{i j}$ are the components of, dielectric and magnetic permittivity coefficients; furthermore, $e_{i j}, q_{i j}$ and $d_{i j}$ are the piezoelectric, piezo-magnetic and magneto-electric-elastic coefficients, respectively.

The reduced stiffness modulus of sandwich composite can be expressed by:

$$
\begin{gathered}
Q_{11}=\frac{E_{11}}{1-v_{12} v_{21}}, Q_{12}=\frac{v_{12} E_{22}}{1-v_{12} v_{21}}, Q_{22}=\frac{E_{22}}{1-v_{12} v_{21}}, \\
Q_{44}=G_{23}, Q_{55}=G_{13}, Q_{66}=G_{12}
\end{gathered}
$$

Transformed shell principal coordinates are in expressed Appendix A.

Now via Hamilton's principle can be written:

$$
\int_{0}^{t} \delta(V+U) \mathrm{d} t=0
$$

where, $V$ is the work done by external energy, $U$ is strain energy. 
The strain energy is expressed as:

$$
\begin{aligned}
U= & \frac{1}{2} \sum_{n=1}^{N} \int_{0}^{a} \int_{0}^{b} \int_{h_{n-1}}^{h_{n}}\left[\left(\sigma_{x x} \varepsilon_{x x}+\sigma_{y y} \varepsilon_{y y}+\tau_{y z} \gamma_{y z}+\tau_{x z} \gamma_{x z}+\tau_{x y} \gamma_{x y}-D_{x} \delta E_{x}\right.\right. \\
& \left.-D_{y} \delta E_{y}\left(-D_{z} \delta E_{z}-B_{x} \delta Q_{x}-B_{y} \delta Q_{y}-B_{z} \delta Q_{z}\right)\right] \\
& \times\left(1+\frac{z}{R_{1}}\right)\left(1+\frac{z}{R_{2}}\right) \mathrm{d} c_{1} \mathrm{~d} c_{2} \mathrm{~d} z
\end{aligned}
$$

The first variation can be obtained as:

$$
\begin{aligned}
\delta U= & \sum_{n=1}^{N} \int_{0}^{a} \int_{0}^{b}\left[\left(N_{x x} \delta \varepsilon_{x x}^{0}+M_{x x} \delta k_{x x}^{0}+P_{x x} \delta k_{x x}^{2}+N_{y y} \delta \varepsilon_{y y}^{0}+M_{y y} \delta k_{y y}^{2}+P_{y y} \delta k_{y y}^{2}\right.\right. \\
& +N_{x y} \delta \varepsilon_{x y}^{0}+M_{x y} \delta k_{x y}^{2}+P_{x y} \delta k_{x y}^{2}+K_{y y} \delta \gamma_{y z}^{0}++R_{y y} k_{y z}^{1}+K_{x x} \delta \gamma_{x z}^{0}+R_{x x} k_{x z}^{1} \\
& \times\left(1+\frac{z}{R_{1}}\right)\left(1+\frac{z}{R_{2}}\right) \mathrm{d} c_{1} \mathrm{~d} c_{2}+\int_{0}^{a} \int_{0}^{b} \int_{-h / 2}^{h / 2}\left[-D_{x} \cos (\xi z) \frac{\partial \delta \phi}{\partial x}-D_{y} \cos (\xi z) \frac{\partial \delta \phi}{\partial y}\right. \\
& +D_{z} \xi \sin (\xi z) \delta \phi-B_{x} \cos (\xi z) \frac{\partial \delta \psi}{\partial x}-B_{y} \cos (\xi z) \frac{\partial \delta \psi}{\partial y} \\
& \left.+B_{z} \xi \sin (\xi z) \delta \varphi \psi\right] \mathrm{d} z \mathrm{~d} x \mathrm{~d} y
\end{aligned}
$$

where, for convenience a shell by rectangular base in dimension a and $\mathrm{b}$ in $c_{1}$ and $c_{2}$ directions, has been considered.

$q_{1}, q_{2}$ are the Lame coefficients of the shell can be expressed as $q_{1}=c_{1}\left(1+\frac{Z}{R_{1}}\right), q_{2}=c \cdot 2$ $\left(1+\frac{Z}{R_{2}}\right)$.

$R_{1}$ and $R_{2}$ are the principal radii of curvature in $q_{1}$ and $q_{1}$ directions, respectively.

The first variation of work can be expressed in the following form:

$$
\delta \Pi_{w}=\int_{0}^{2 \pi} \int_{0}^{L}\left[N_{x}^{0} \frac{\partial w_{0}}{\partial x} \frac{\partial \delta w_{0}}{\partial x}\right] \mathrm{d} c_{1} \mathrm{~d} c_{2}
$$

It is assumed that the composite doubly curved shell is exposed to external electric voltage $V$, magnetic potential $\Omega$ and variation of temperature and moisture applied forces. $N_{x}^{0}$ can be written as:

$$
N_{x}^{0}=N^{E}+N^{Q}+N^{T}+N^{H}
$$

where:

$$
\begin{aligned}
& N^{E}=-\int_{-h / 2}^{h / 2} e_{31} \frac{2 V}{h} \mathrm{~d} z, \\
& N^{Q}=-\int_{-h / 2}^{h / 2} q_{31} \frac{2 \Omega}{h} \mathrm{~d} z,
\end{aligned}
$$




$$
\begin{aligned}
& N^{T}=\int_{h_{n-1}}^{h_{n}}\left[\bar{Q}_{11} \alpha_{11}+\bar{Q}_{12} \alpha_{12}\right]\left(\mathrm{T}-\mathrm{T}_{1}\right) \mathrm{d} z, \\
& N^{H}=\int_{h_{n-1}}^{h_{n}}\left[\bar{Q}_{11} \beta_{11}+\bar{Q}_{12} \beta_{12}\right]\left(H-H_{1}\right) \mathrm{d} z
\end{aligned}
$$

and $T-T_{1}, H-H_{1}$ are variation of temperature and moisture, $T$ can be defined by sinusoidal temperature following as:

$$
T=T_{1}+\Delta T\left(1-\operatorname{Cos} \frac{\pi}{2}\left(\frac{1}{2}+\frac{z}{h}\right)\right)
$$

By setting the coefficients of $\delta u, \delta v, \delta w, \delta \varphi_{x}, \delta \varphi_{y}, \delta \phi$ and $\delta \psi$ to zero and substituting Eqs. (26) and (30a, 30b, 30c, 30d) into Eq. (25) may be stated as:

$$
\begin{gathered}
\frac{\partial N_{x}}{\partial x}+\frac{\partial N_{x y}}{\partial y}=0 \\
\frac{\partial N_{x y}}{\partial x}+\frac{\partial N_{y}}{\partial y}=0 \\
\frac{\partial \bar{K}_{x}}{\partial x}+\frac{\partial \bar{K}_{y}}{\partial y}+\frac{\partial}{\partial x}\left[N_{x}\left(\frac{\partial w_{0}}{\partial x}-\frac{u_{0}}{R_{1}}\right)\left[+N_{x y}\left(\frac{\partial w_{0}}{\partial y}-\frac{v_{0}}{R_{2}}\right)\right]\right. \\
+\frac{\partial}{\partial y}\left[N_{x y}\left(\frac{\partial w_{0}}{\partial x}-\frac{u_{0}}{R_{1}}\right)+N_{y}\left(\frac{\partial w_{0}}{\partial y}-\frac{v_{0}}{R_{2}}\right)\right]+s_{1}\left(\frac{\partial^{2} P_{y}}{\partial x^{2}} l l+2 \frac{\partial^{2} P_{x y}}{\partial x \partial y}+\frac{\partial^{2} P_{y}}{\partial y^{2}}\right)-\frac{N_{x}}{R_{1}} \\
-\frac{N_{y}}{R_{2}}+q^{\text {hyg }}=0 l l
\end{gathered}
$$

where:

$$
\begin{gathered}
\bar{M}_{i}=M_{i}-s_{1} P_{i}(i=1,2,6), s_{1}=\frac{4}{3 h^{2}}, s_{2}=3 s_{1} \\
\bar{K}_{j}=K_{j}-s_{2} R_{j}(j=1,2)
\end{gathered}
$$




\section{Solution procedure}

The boundary conditions of the multiscale composite have been considered simply-supported $(\mathrm{S}-\mathrm{S})$ :

$$
\begin{gathered}
u_{0}(x, 0, t)=u_{0}(x, b, t)=0, v_{0}(0, y, t)=v_{0}(x, b, t)=0, \\
w_{0}(x, 0, t)=w_{0}(x, b, t)=0 \\
\varphi_{y}(0, y, t)=\varphi_{y}(a, y, t)=0, \varphi_{x}(x, 0, t)=\varphi_{x}(x, b, t)=0, \\
N_{x}(x, 0, t)=N_{x}(x, b, t)=0, N_{y}(0, y, t)=N_{y}(a, y, t)=0, \\
\bar{M}_{x}(x, 0, t)=\bar{M}_{x}(x, b, t)=0, \bar{M}_{y}(0, y, t)=\bar{M}_{y}(a, y, t)=0 .
\end{gathered}
$$

Furthermore for obtaining the boundary conditions, the displacement of the composite shell is given as:

$$
\begin{aligned}
u(x, y, t) & =\sum_{n=1}^{\infty} \sum_{m=1}^{\infty} U_{m n}(t) \cos \left(\frac{n \pi x}{b}\right) \sin (m y), \\
v_{0}(x, y, t) & =\sum_{n=1}^{\infty} \sum_{m=1}^{\infty} V_{m n}(t) \cos \left(\frac{n \pi x}{b}\right) \sin (m y), \\
w_{0}(x, y, t)= & \sum_{n=1}^{\infty} \sum_{m=1}^{\infty} W_{m n}(t) \cos \left(\frac{n \pi x}{b}\right) \sin (m y), \\
\varphi_{x}(x, y, t)= & \sum_{n=1}^{\infty} \sum_{m=1}^{\infty} \varphi_{x m n}(t) \cos \left(\frac{n \pi x}{b}\right) \sin (m y), \\
\varphi_{y}(x, y, t)= & \sum_{n=1}^{\infty} \sum_{m=1}^{\infty} \varphi_{y m n}(t) \cos \left(\frac{n \pi x}{b}\right) \sin (m y), \\
\phi(x, y, t)= & \sum_{n=1}^{\infty} \sum_{m=1}^{\infty} \phi_{m n}(t) \cos \left(\frac{n \pi x}{b}\right) \sin (m y), \\
\psi(x, y, t)= & \sum_{n=1}^{\infty} \sum_{m=1}^{\infty} \psi_{m n}(t) \cos \left(\frac{n \pi x}{b}\right) \sin (m y)
\end{aligned}
$$

where $U_{m n}(t), V_{m n}(t), W_{m n}(t), \varphi_{x m n}(t), \varphi_{y m n}(t), \phi(t)$ and $\psi(t)$ refer to the unknown functions of the time; $n$ and $m$ are the number mode of frequency in the $x$ and $y$ directions, respectively. 
Here, $\frac{n \pi x}{b}=l$ are assumed.

By substituting Eq. (35) into Eq. (32) and driving the Navier procedure, the following expressions can be expressed:

$$
\begin{aligned}
& a_{11} U_{m n}(t)+ a_{12} V_{m n}(t)+a_{13} W_{m n}(t)+a_{14} \varphi_{x m n}(t)+a_{15} \varphi_{y m n}(t)+a_{16} \phi_{m n}(t) \\
&+a_{17} \psi_{m n}(t)=M_{11} \ddot{U}_{m n}(t)+M_{13} \ddot{W}_{m n}+M_{14} \ddot{\varphi}_{x m n}(t), \\
& a_{21} U_{m n}(t)+ a_{22} V_{m n}(t)+a_{23} W_{m n}(t)+a_{24} \varphi_{x m n}(t)+a_{25} \varphi_{y m n}(t)+a_{26} \phi_{m n}(t) \\
&+a_{27} \psi_{m n}(t)=M_{22} \ddot{V}_{m n}(t)+M_{23} \ddot{W}_{m n}+M_{25} \ddot{\varphi}_{y m n}(t), \\
& a_{31} U_{m n(t)}+a_{32} V_{m n}(t)+a_{33} W_{m n}(t)+a_{34} W_{m n}^{3}(t)+a_{35} \varphi_{x m n}(t)+a_{36} \varphi_{y m n}(t) \\
&+a_{37} \phi_{m n}(t)+a_{38} \psi_{m n}(t)=M_{33} \ddot{W}_{m n}+M_{34} \ddot{\varphi}_{x m n}(t)+M_{35} \ddot{\varphi}_{y m n}(t), \\
& a_{41} U_{m n}(t)+a_{42} V_{m n}(t)+a_{43} W_{m n}(t)+a_{44} \varphi_{x m n}(t)+a_{45} \varphi_{y m n}(t)+a_{46} \phi_{m n}(t) \\
&+a_{47} \psi_{m n}(t)=M_{44} \ddot{\varphi}_{x m n}(t), \\
& a_{51} U_{m n}(t)+a_{52} V_{m n}(t)+a_{53} W_{m n}(t)+a_{54} \varphi_{x m n}(t)+a_{55} \varphi_{y m n}(t)+a_{56} \phi_{m n}(t) \\
&+a_{57} \psi_{m n}(t)=0, \\
& a_{61} U_{m n}(t)+a_{62} V_{m n}(t)+a_{63} W_{m n}(t)+a_{64} \varphi_{x m n}(t)+a_{65} \varphi_{y m n}(t)+a_{66} \phi_{m n}(t) \\
&+a_{67} \psi_{m n}(t)=0, \\
&+a_{72} V_{m n}(t)+a_{73} W_{m n}(t)+a_{74} \varphi_{x m n}(t)+a_{75} \varphi_{y m n}(t)+a_{76} \phi_{m n}(t) \\
&+ a_{77} \psi_{m n}(t)=0
\end{aligned}
$$

where the coefficients $a_{i j}$ and $M_{i j}$ experssion stiffness matrix and mass matrix of sandwich composite shell that are defined in Appendix.

The non-linear differential equation of nano composite can be driven as:

$$
\frac{d^{2} W_{m n}(t)}{\mathrm{d} t^{2}}+P_{1} W_{m n}(t)+P_{2} W_{m n}^{2}(t)+P_{3} W_{m n}^{3}(t)=0
$$

where initial conditions are illustrated by:

$$
W_{m n}(0)=\frac{\bar{W}}{h},\left.\frac{\mathrm{d} W_{m n}(t)}{\mathrm{d} t}\right|_{t=0}=0
$$

To study the bifurcations of the established non-linear system, the method of multiscale is a powerful tool to determine the solutions for conservative and non-conservative systems. Hence, this method is adopted to investigate the post buckling responses of the composite doubly curved shell.

The uniformly approximate solutions of (37) are obtained as:

$$
w=w_{0}\left(T_{0}, T_{1}, T_{2}, \ldots\right)+\varepsilon w_{1}\left(T_{0}, T_{1}, T_{2}, \ldots\right)+\varepsilon^{2} w_{2}\left(T_{0}, T_{1}, T_{2}, \ldots\right)
$$

where $T_{0}=t$ and $T_{1}=\varepsilon t$. 
Then, the derivatives with respect to $t$ become:

$$
\begin{gathered}
\frac{d}{\mathrm{~d} t}=D_{0}+\varepsilon D_{1}, \\
\frac{d}{\mathrm{~d} t}=D_{0}^{2}+2 \varepsilon D_{0} D_{1}+\varepsilon^{2}\left(D_{1}^{2}+2 D_{0} D_{1}\right)
\end{gathered}
$$

Substituting (43) and (44) into (41) and equating the coefficients of $\varepsilon$ to zero yield the following differential equations:

$$
\begin{gathered}
\varepsilon^{0}: D_{0}^{2} w_{0}+w_{0}=0 \\
\varepsilon^{1}: D_{0}^{2} w_{1}+\Omega^{2} w_{1}=-2 D_{0} D_{1} w_{0}-\mu D_{0} w_{0}-P_{3} w_{0}^{3}
\end{gathered}
$$

With this approach it turns out to be convenient to write the solution of Eqs. (42-43) as:

$$
w_{0}\left(T_{0}, T_{1}, T_{2}, \ldots\right)=\exp \left(i T_{0}\right)+\bar{A} \exp \left(-i T_{0}\right)
$$

where $A$ is an unknown complex function and $A$ is the complex conjugate of $A$. The governing equations for $A$ are obtained by requiring $w_{1}$ to be periodic in $T_{0}$ and extracting secular terms the solvability equation will be determined as:

$$
2 i w_{0}\left(A^{\prime}+\mu A\right)+3 P_{3} A^{2} \overline{\mathrm{A}}=0
$$

Let $A$ be in polar form:

$$
A=\frac{1}{2} a \exp (i \gamma)
$$

\section{Results and discussion}

This section elucidates the numerical results of the postbuckling behaviour of multiscale piezoelectric doubly curved shells under the influence of external hygrothermal loads (Fig. 1). The material properties corresponding to the multiscale composite shell and piezoelectric layer are tabulated in Tables 1 and 2, respectively. The present study considers Elliptic paraboloid shell $\left(R_{1} \neq R_{2}\right)$ for the evaluation. The multiscale reinforcements such as carbon nanotube and graphene platelet with effective thickness $t_{\mathrm{cnt}}=0.0348 \mathrm{~nm}$ and $t_{\mathrm{gpl}}=$ $0.0348 \mathrm{~nm}$, respectively, are selected. For calculations, $\mathrm{G}_{13}=\mathrm{G}_{23}=0.5 \mathrm{G}_{12}$ is considered. To justify the credibility of the proposed formulation to predict accurate buckling characteristics, the results from this study are compared with the previously published results of Shen and Zhang [85] and Mayandi and Jeyaraj [86]. The verification study evaluates the critical buckling of square plate for $U, A, X, O$ distribution pattern with $R_{2}=\infty$ and $\frac{b}{h}=20$. In addition, a higher order shear deformation theory is assumed. From Table 3 it is affirmed that the results are matching very well with that of Shen and Zhang [85] and Mayandi and Jeyaraj [86]. Therefore, this formulation can be incorporated to assess the postbuckling behaviour of multiscale piezoelectric composite shells effectively. 


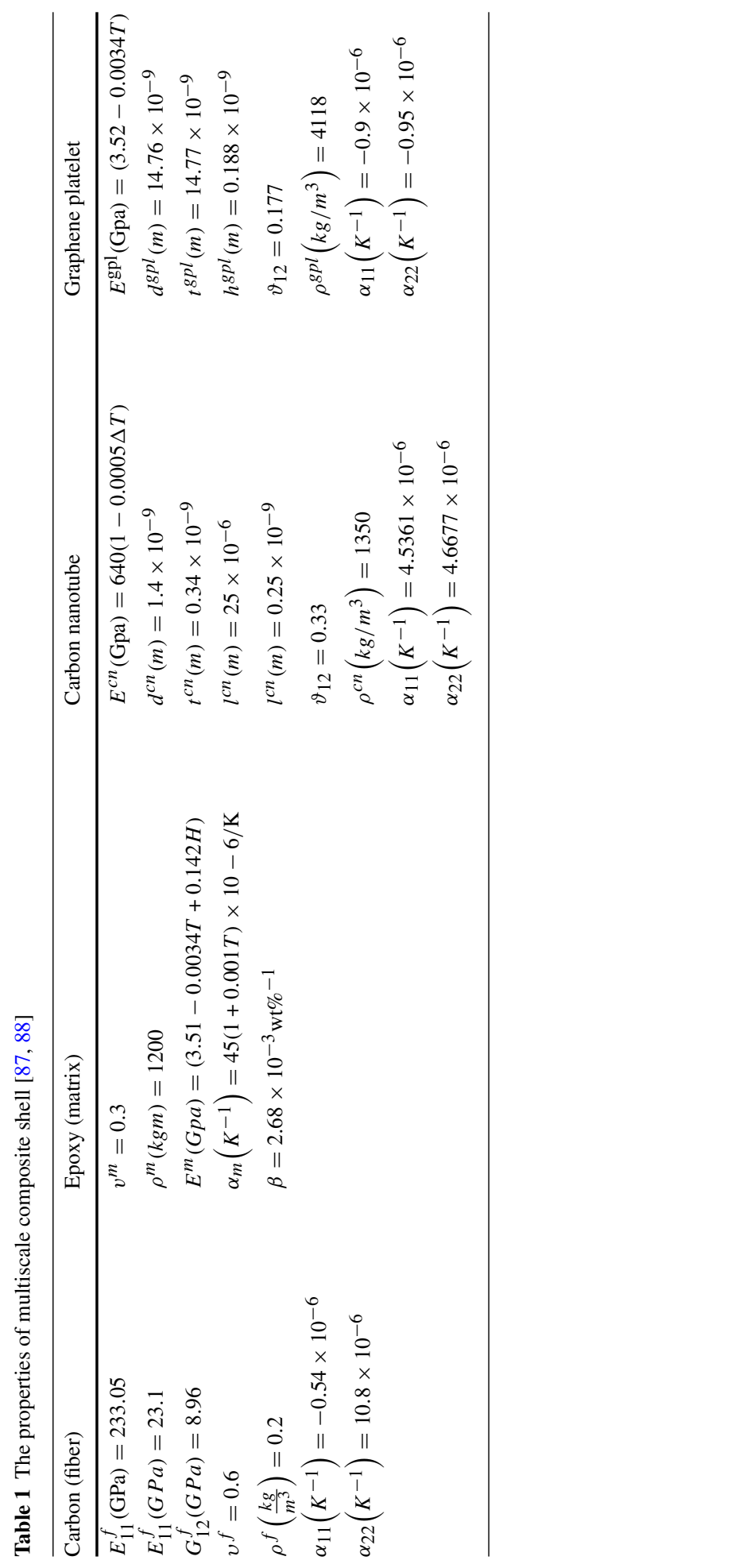




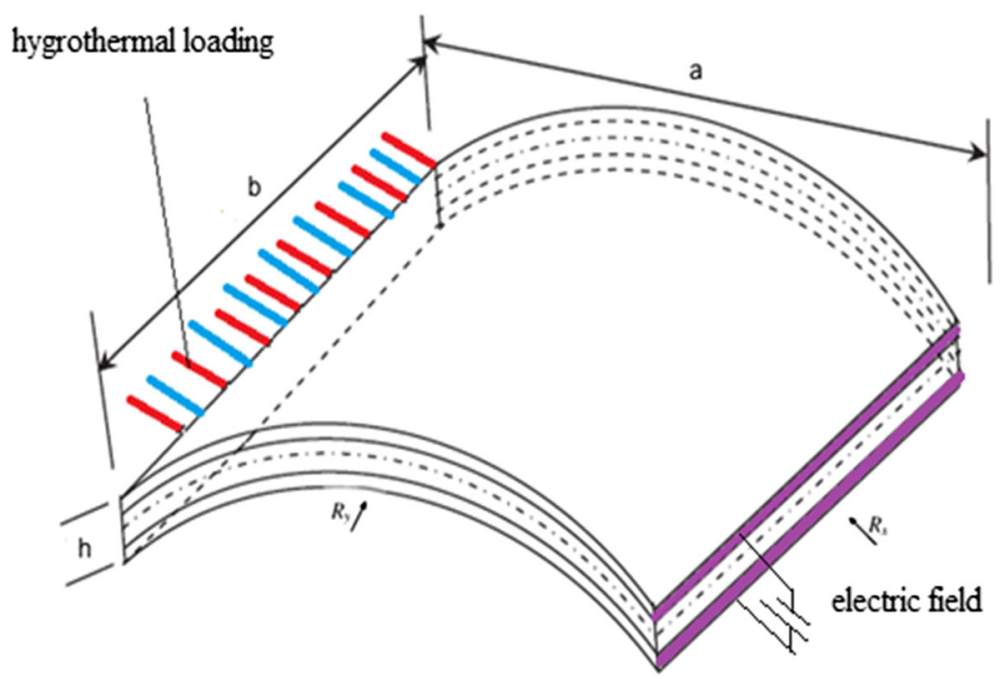

Fig. 1 Geometry of doubly curved multiscale composite shell in hygrothermal environment with piezoelectric layer

Table 2 Material properties of $\mathrm{BiTiO}_{3}-\mathrm{CoFe}_{2} \mathrm{O}_{4}$ [89-92]

\begin{tabular}{ll}
\hline Properties $\mathrm{BiTiO}_{3}-\mathrm{CoFe}_{2} \mathrm{O}_{4}$ & \\
\hline Piezoelectric/(C $\left.\cdot \mathrm{m}^{-2}\right)$ & $e_{31}=-2.2, e_{33}=9.3, e_{15}=5.8$ \\
\hline Dielectric/ $\left(10^{-9} \mathrm{C} \cdot \mathrm{V}^{-1} \cdot \mathrm{m}^{-1}\right)$ & $d_{11}=5.64, d_{33}=6.35$ \\
Piezomagnetic/(N $\left.\cdot \mathrm{A}^{-1} \cdot \mathrm{m}-1\right)$ & $q_{15}=275, q_{31}=290.1, q_{33}=349.9$ \\
Magnetoelectric/ $\left(10^{-12} \mathrm{Ns} \cdot \mathrm{V}^{-1} \cdot \mathrm{C}^{-1}\right)$ & $s_{11}=5.367, s_{33}=2737.5$ \\
\hline
\end{tabular}

Table 3 Comparison of critical buckling load for laminated plate $(b / h=20, a / b=1 ; T=300 \mathrm{~K})$

\begin{tabular}{|c|c|c|c|c|c|c|c|}
\hline \multirow[t]{4}{*}{$\mathrm{V}^{\mathrm{gpl}}$} & \multirow{4}{*}{$\begin{array}{l}\text { Distribution } \\
\text { pattern }\end{array}$} & \multicolumn{6}{|c|}{ Critical buckling load } \\
\hline & & \multicolumn{6}{|l|}{$\mathrm{a} / \mathrm{b}$} \\
\hline & & \multicolumn{3}{|l|}{1} & \multicolumn{3}{|l|}{2} \\
\hline & & $\begin{array}{l}\text { Shen and } \\
\text { Zhang [68] }\end{array}$ & $\begin{array}{l}\text { Mayandi and } \\
\text { Jeyaraj [69] }\end{array}$ & Present & $\begin{array}{l}\text { Shen and } \\
\text { Zhang [68] }\end{array}$ & $\begin{array}{l}\text { Mayandi and } \\
\text { Jeyaraj [69] }\end{array}$ & Present \\
\hline \multicolumn{8}{|l|}{0.12} \\
\hline & $U$ & 340.74 & 344 & 340.005 & 317.52 & 318 & 317.349 \\
\hline & $X$ & 355.85 & 361 & 354.295 & 323.59 & 324 & 323.177 \\
\hline
\end{tabular}

It is worthy to mention at this point that unless and until stated the following parameters are considered for the evaluation. The stacking sequence of cross ply multiscale doubly curved piezoelectric shell (MDCPS) is $\left[0^{\mathrm{PCF}} / 90^{\mathrm{PGF}}\right]_{\mathrm{S}}, \frac{a}{R_{1}}=0.1, \frac{b}{R_{2}}=0.05, h=2 \mathrm{~mm}, \frac{a}{h}=$ $10, \frac{a}{b}=1, T=300, \Delta H=1, V=50 ; \Omega=0.2$ and $(m, n=1,2)$.

The effect of externally applied electric voltage on the hygrothermal postbuckling behaviour of MDCPS is investigated. Figure 2 illustrates the hygrothermal postbuckling 


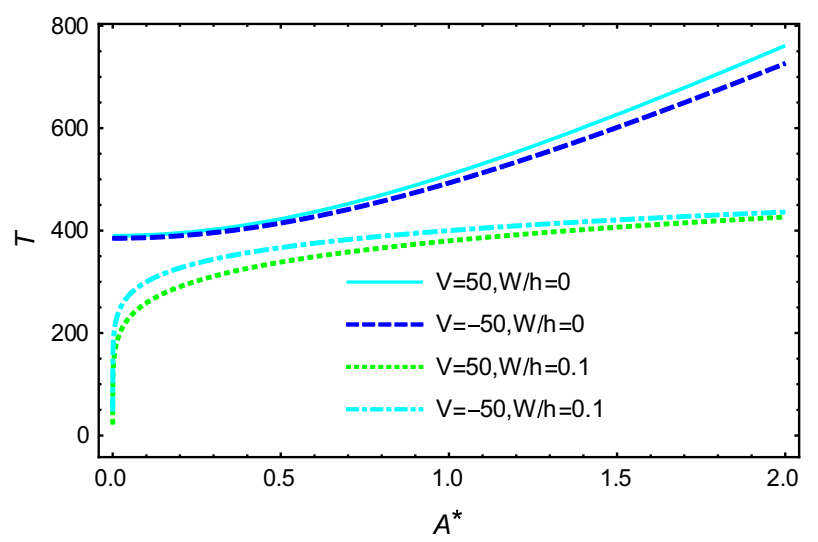

Fig. 2 Influence of various applied voltage versus thermal postbuckling load-deflection curve of MDCPS with $\frac{a}{R_{1}}=0.1, \frac{b}{R_{2}}=0.05, h=2 \mathrm{~m}, \Omega=0.2, H=1$ and $(m, n=1,2)$

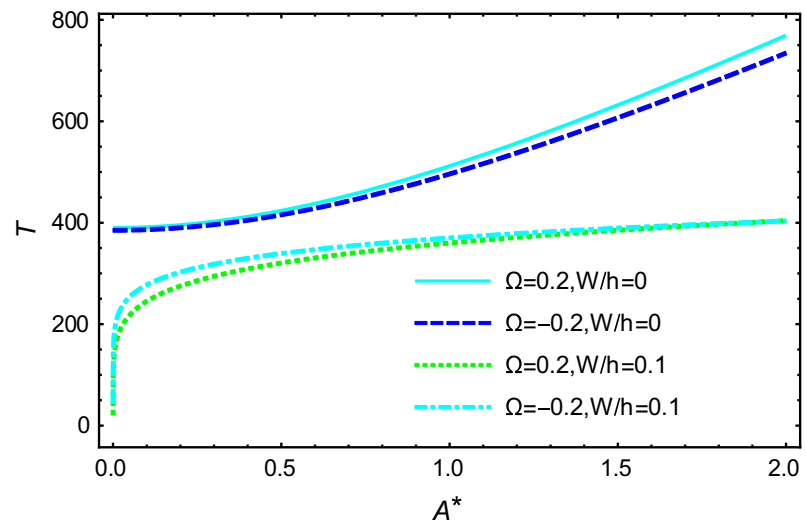

Fig. 3 Influence of various magnetic potential to thermal postbuckling load-deflection curve of MDCPS with $\frac{a}{R_{1}}=0.1, \frac{b}{R_{2}}=0.05, h=2 \mathrm{~m}, V=50, H=1$ and $(m, n=1,2)$ for $A$ distribution pattern

load-deflection curve with different magnitudes of electric voltage ( $-50 \mathrm{~V}, 0,50 \mathrm{~V})$. It can be witnessed from this figure that an increment in the temperature results in consistently raising hygrothermal postbuckling load-deflection curve. However, the influence of temperature gradient is minimal. In addition, a higher magnitude of external voltage has a significant influence. It is observed that positive applied voltage has more effect for $W / h=0$ whereas, a negative voltage displays a predominant influence on the postbuckling load-deflection curve for $W / h=0.1$.

Figure 3 explains the influence of external magnetic field on the variation of hygrothermal postbuckling load-deflection curve of MDCPS. In this case the voltage applied is restricted to $50 \mathrm{~V}$. Meanwhile two different magnitudes of magnetic potential viz. 0.2 and -0.2 are considered for analysis. It can be witnessed from this figure that as the temperature improves the hygrothermal postbuckling load-deflection curve also shows an increment. Similar to the effect of electric voltage, for $W / h=0$ and $W / h=0.1$ a significant influence of positive and negative magnetic potentials is observed, respectively. 


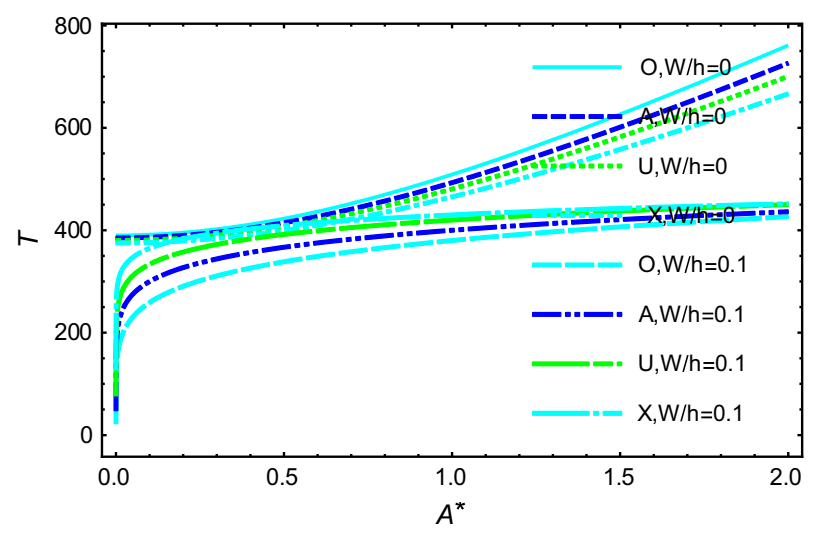

Fig. 4 Influence of different distribution pattern versus thermal postbuckling load-deflection curve of MDCPS with $\frac{a}{R_{1}}=0.1, \frac{b}{R_{2}}=0.05, h=2 \mathrm{~mm}, T=300, \Delta H=1$ and $(m, n=1,2)$

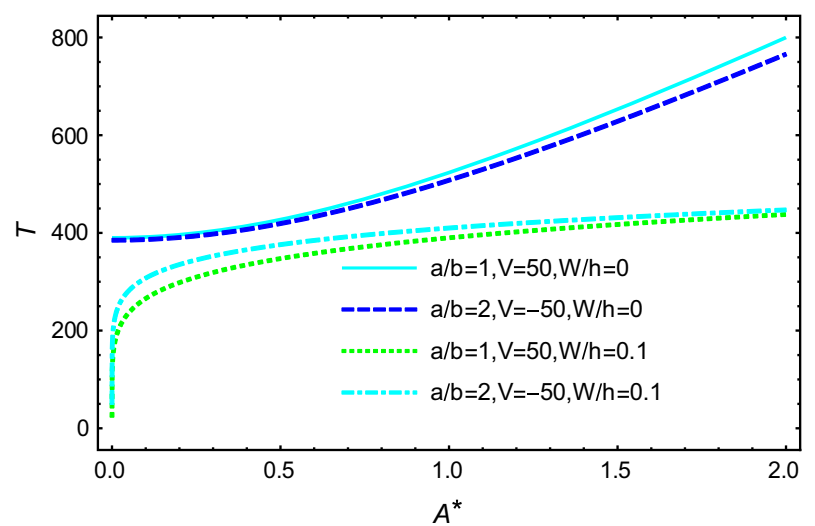

Fig. 5 Influence of aspect ratio versus non-linear to thermal postbuckling load-deflection curve of MDCPS with $\frac{a}{R_{1}}=0.1, \frac{b}{R_{2}}=0.05, h=2 \mathrm{~mm}, T=300, \Delta H=1$ and $(m, n=1,2)$

The influence of various distribution patterns of multiscale reinforcements such as $X, A, U$, $O$ on the hygrothermal postbuckling behaviour of MDCPS is studied and illustrated in Fig. 4. The results reveal that the load-deflection curve exhibited a higher value for $O$ distribution whereas a minimum value is noticed for $X$ distribution. This trend holds good for both $\mathrm{W} / \mathrm{h}$ $=0$ and $W / h=0.1$

The effect of aspect ratio and electric voltage together on the hygrothermal postbuckling load-deflection curve of MDCPS is shown in Fig. 5. It is clear that aspect ratio is the important factor to study thin curved panel structures. Further, for $W / h=0$, higher aspect ratio has an adverse effect on the load-deflection curve whereas for $W / h=0.1$, improving the aspect ratio leads to improved thermal postbuckling load-deflection curve.

A similar analysis is extended to assess the influence of aspect ratio associated with the magnetic potential on the hygrothermal postbuckling load-deflection curve of MDCPS, as depicted in Fig. 6. Via numerical results, it is observed that for both $W / h=0$ and $W / h=0.1$, increasing the aspect ratio and magnetic potential on thermal postbuckling load-deflection 


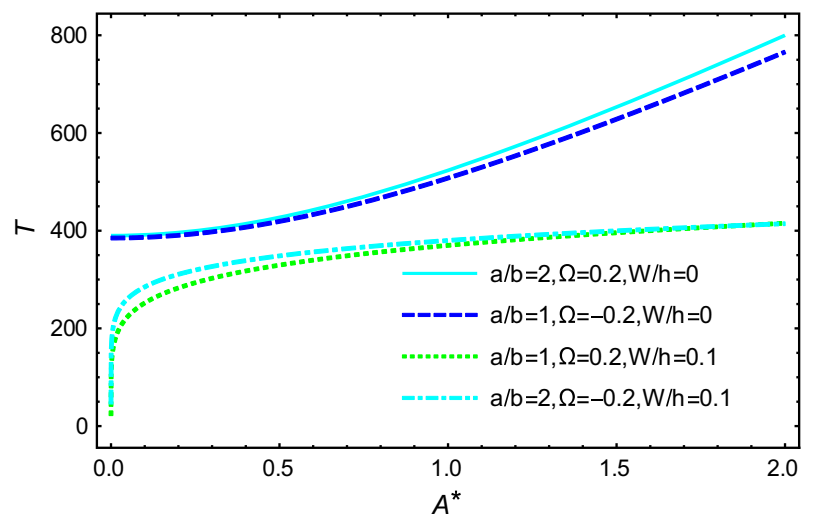

Fig. 6 Influence of aspect ratio on thermal postbuckling load-deflection curve of MDCPS with $\frac{a}{R_{1}}=$ $0.1, \frac{b}{R_{2}}=0.05, h=2 \mathrm{~mm}, T=300, \Delta H=1$ and $(m, n=1,2)$

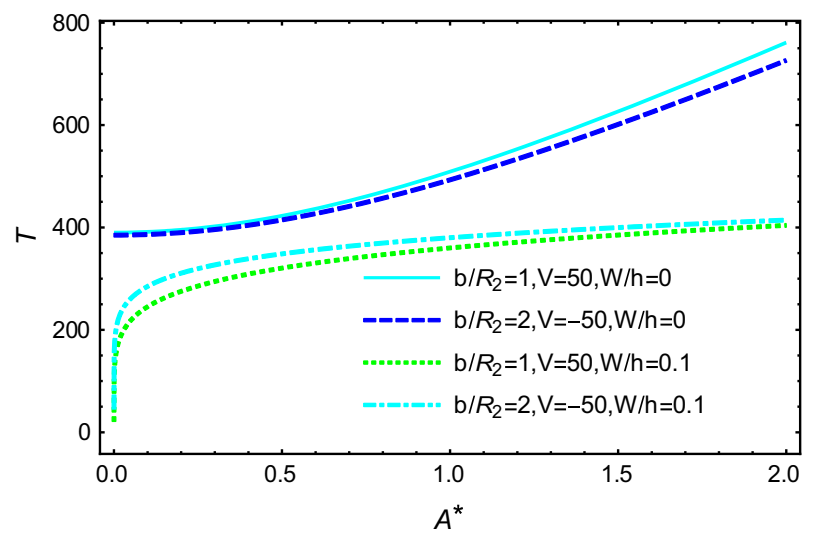

Fig. 7 Influence of curvature ratio versus on thermal postbuckling load-deflection curve of MDCPS with applied electric voltage $\left(\frac{a}{R_{1}}=0.1, \frac{b}{R_{2}}=0.05, h=2 \mathrm{~mm}, T=300, \Delta H=1\right.$ and $\left.m, n=1,2\right)$

curve increases and the significant influence is noticed for the positive magnitude of magnetic potential.

Influence of curvature ratio $\left(\frac{b}{R_{2}}\right)$ and applied voltage with positive and negative magnitude under on thermal postbuckling load-deflection curve of MDCPS is presented in Fig. 7. It is found that increasing of curvature value lead to decrease on the thermal postbuckling load-deflection curve and positive magnitude of applied voltage has the most effect on curve while, the negative applied voltage has the lowest magnitude.

Analogously, the effect of curvature ratio $\left(\frac{b}{R_{2}}\right)$ and magnetic potential on the buckling characteristics is studied as well (Fig. 8). From this figure, it can be witnessed that curvature ratio has reducer effect on thermal postbuckling load-deflection curve for $W / h=0$. Furthermore, negative magnetic potential has lower influence on non-linear amplitude curve than positive magnetic potential magnitude. Meanwhile, for $W / h=0.1$, a reverse trend is noticed. 


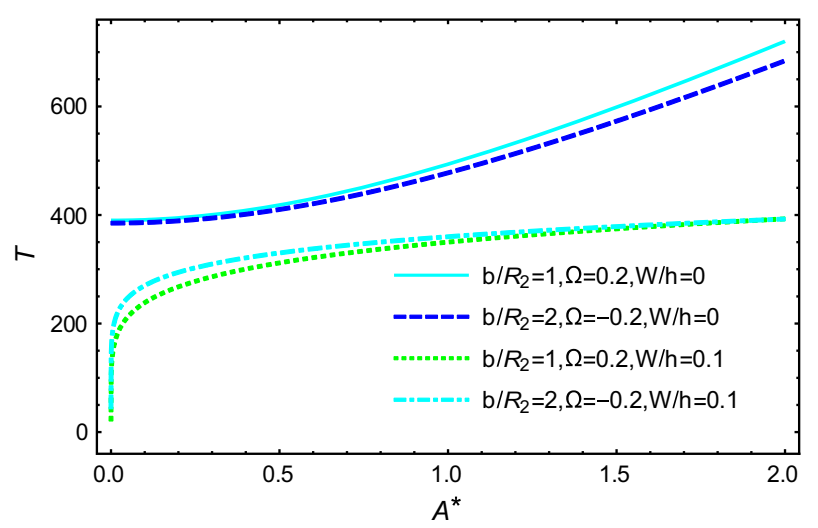

Fig. 8 Influence of curvature ratio versus on thermal postbuckling load-deflection curve of MDCPS with applied magnetic potential $\left(\frac{a}{R_{1}}=0.1, \frac{b}{R_{2}}=0.05, h=2 \mathrm{~mm}, T=300, \Delta H=1\right.$ and $\left.m, n=1,2\right)$

\section{Conclusion}

This article studies the hygrothermal postbuckling characteristics of multiscale composite doubly curved shell through analytical approach. In this regard, Halpin-Tsai model is incorporated along with Von Karman type geometric non-linearity. The kinematics of MDCPS is assumed to follow third-order shear deformation theory. The governing equations of motion are derived through Hamilton's principle. The solution to the postbuckling problem of MDCPS is obtained using the multiple scale method. The parametric study is performed to assess the influence of temperature and moisture gradient, distribution patterns of GPL and CNT, external applied voltage and magnetic potentials, aspect ratio and curvature ratio. The numerical results reveal that the load-deflection curve improves with higher hygrothermal loading. Further, the positive electric voltage and magnetic potential has a significant influence on the load-deflection curve. Also, the curvature ratio has reducer effect on thermal postbuckling load-deflection curve.

\section{Appendix}

Transformed shell principle coordinate can be expressed by:

$$
\begin{aligned}
& \bar{Q}_{11}^{n}=Q_{11}^{n} \cos ^{4} \theta+2\left(Q_{12}^{n}+2 Q_{66}^{n}\right) \sin ^{2} \theta \cos ^{2} \theta+Q_{22}^{n} \sin ^{4} \theta \\
& \bar{Q}_{12}^{n}=\left(Q_{11}^{n}+Q_{22}^{n}-4 Q_{66}^{n}\right) \sin ^{2} \theta \cos ^{2} \theta+Q_{12}^{n}\left(\sin ^{4} \theta+\cos ^{4} \theta\right) \\
& \bar{Q}_{22}^{n}=Q_{11}^{n} \sin ^{4} \theta+2\left(Q_{12}^{n}+2 Q_{66}^{n}\right) \sin ^{2} \theta \cos ^{2} \theta+Q_{22}^{n} \cos ^{4} \theta \\
& \bar{Q}_{66}^{n}=\left(Q_{11}^{n}+Q_{22}^{n}-2 Q_{12}^{n}-2 Q_{66}^{n}\right) \sin ^{2} \theta \cos ^{2} \theta+Q_{66}^{n}\left(\sin ^{4} \theta+\cos ^{4} \theta\right) \\
& \bar{Q}_{44}^{n}=Q_{44}^{n} \cos ^{2} \theta+Q_{55}^{n} \sin ^{2} \theta \\
& \bar{Q}_{55}^{n}=Q_{55}^{n} \cos ^{2} \theta+Q_{44}^{n} \sin ^{2} \theta
\end{aligned}
$$

\section{References}

1. G.M. Odegard, S.J.V. Frankland, T.S. Gates, AIAA J. 43(8), 1828 (2005) 
2. X.L. Gao, K. Li, Int. J. Solids. Struct. 42(5), 1649-1667 (2005)

3. W.Q. Chen, J.B. Cai, G.R. Ye, AIAA J. 41(11), 2244-2250 (2003)

4. N.S. Naidu, P.K. Sinha, Compos. Struct. 77(4), 475-483 (2007)

5. M. Amabili, J.N. Reddy, J.N. Int, J. Nonlin. Mech. 45(4), 409-418 (2010)

6. V.K. Singh, S.K. Panda, Thin Wall. Struct. 85, 341-349 (2014)

7. S.M. Chorfi, A. Houmat, Compos. Struct. 92(10), 2573-2581 (2010)

8. F. Alijani, M. Amabili, K. Karagiozis, F. Bakhtiari-Nejad. J. Sound Vib. 330(7), 1432-1454 (2011)

9. H.S. Shen, X. Chen, L. Guo, L. Wu, X.L. Huang, X. L, Aerosp. Sci. Technol. 47, 434-446 (2015).

10. K.M. Saad, O.S. Iyiola, P. Agarwal, AIMS Math. 3(1), 183-194 (2018)

11. A.A. Yazdi, Compos. Struct. 96, 526-531 (2013)

12. S.K. Panda, B.N. Singh, Aerosp. Sci. and Technol. 29(1), 47-57 (2013)

13. S. Pradyumna, A. Gupta, A. Acta mech. 218(3-4), 295-308 (2011)

14. Y. Fan, H. Wang, Nonlinear Dyn. 1-14 (2017).

15. M.M. Heydari, A.H. Bidgoli, H.R. Golshani, G. Beygipoor, A. Davoodi, Nonlinear Dyn. 79(2), 1425-1441 (2015)

16. M. Rafiee, M. Mohammadi, B.S. Aragh, H. Yaghoobi, H. Compos, Struct. 103, 179-187 (2013)

17. S. Razavi, A. Shooshtari, Compos Struct. 119, 377-384 (2015)

18. H.S. Shen, F. Lin, Y. Xiang, Nonlinear Dyn. 90(2), 899-914 (2017)

19. F. El-Haina, A. Bakora, A.A. Bousahla, A. Tounsi S.R. Mahmoud, Struct. Eng. Mech. 63(5), 585-595 (2017).

20. A. Menasria, A. Bouhadra, A. Tounsi, A.A. Bousahla, S.R. Mahmoud, Steel Compos. Struct. 25(2), 157-175 (2017)

21. A. Attia, A.A. Bousahla, A. Tounsi, S.R. Mahmoud A. S. Alwabli, Struct. Eng. Mech. 65(4) $453-464$ (2018).

22. A. Chikh, A. Tounsi, H. Hebali S. R. Mahmoud, Smart Struct. Syst. 19(3), 289-297 (2017).

23. Y. Beldjelili, A. Tounsi S.R. Mahmoud, Smart Struct. Syst. 18(4), 755-786 (2016).

24. A. Hamidi, M.S.A Houari, S.R. Mahmoud A. Tounsi, Steel Compos. Struct. 18(1), 235 - 253 (2015).

25. A. Tounsi M.S.A. Houari, S Benyoucef, E.A.A.Bediaa, Aerosp. Sci. Technol. 24(1), 209-220 (2013).

26. B. Bouderba, M.S.A. Houri, A. Tounsi, Steel Compos. Struct. 14(1), 85-104 (2013)

27. M. Zidi, A. Tounsi, M.S.A. Houari, E.A.A. Bedia, O.A. Beg, Aerosp. Sci. Tech. 34, 24-34 (2014)

28. A.A. Bousahla, S. Benyoucef, A. Tounsi, S.R. Mahmoud, Struct. Eng. Mech. 60(2), 313-335 (2016)

29. A Besseghier, M Houari, A. Tounsi, S.R. Mahmoud, Smart Struct. Syst., Int. J. 19(6), 601-614 (2017).

30. R. Hamza-Cherif, M. Meradjah, M. Zidour, A. Tounsi, S. Belmahi, T. Bensattalah, J. Nano. Res. 54, 1-14 (2018)

31. H. Khetir, M.B. Bouiadjra, M.S.A. Houari, A. Tounsi, S.R. Mahmoud, Struct. Eng. Mech. 64(4), 391-402 (2017)

32. A. Mouffoki, M. Houari A. Tounsi, Smart Struct. Syst. 20(3), 369-383 (2017).

33. H. Abdelaziz, M. Meziane, A. Bousahla, A. Tounsi, S.R. Mahmoud, A.S. Alwabli, Steel Compos. Struct. 25(6), 693-704 (2017)

34. R. Meksi, S. Benyoucef, A. Mahmoudi, J. Sandw. Struct. Mater. 21(2), 727-757 (2019)

35. H. Bellifa, A. Bakora, A. Tounsi, A.A. Bousahla, S.R. Mahmoud, Steel Compos. Struct. 25(3), 257-270 (2017)

36. A. Zine, A. Tounsi, K. Draiche, M. Sekkal, Steel Compos. Struct. 26(2), 125-137 (2018)

37. M.A. Meziane, H. Abdelaziz, A. Tounsi, J. Sandw. Struct. Mater. 16(3), 293-318 (2014)

38. I. Belkorissat, M. A. Houari, A. Tounsi and E. A. Adda Bedia, Steel Compos. Struct. 18(4), 1063-1081 (2015).

39. A. Younsi, A. Tounsi, F.Z. Zaoui, A.A. Bousahla, S.R. Mahmoud, Geomech. Eng. 14(6), 519-532 (2018)

40. M. Bennoun, M.A. Houari, A. Tounsi, Mech. Adv. Mater. Struct. 23(4), 423-431 (2016)

41. M. Bourada, K. Abdelhakim, M.A. Houari, A. Tounsi, Steel Compos. Struct. 18(2), 409-423 (2015)

42. M Abualnour, M. A. Houari, A. Tounsi, E.A. Adda Bedia, S. R. Mahmoud Compos Struct. 184, 688-697 (2018).

43. A. Bousahla, M. A. Houari, A. Tounsi, E. A. Adda Bedia, Int J Comput. Meth. 11(6), 1350082 (2014).

44. M. Khiloun, A. A. Bousahla, A. Kaci, A. Bessaim, A. Tounsi, S. R. Mahmoud, Eng. Comput. 1-15. (2019).

45. H. Habib, A. Tounsi, M. A. Houari, A. Bessaim, J. Eng. Mech. ASCE. 140, 374-383 (2014).

46. Z. Belabed, M. A. Houari, A. Tounsi, S. R. Mahmoud, O. Anwar Bég, Compos. Part B Eng. 60, 274-283 (2014).

47. K. Draiche, A. Tounsi, S.R. Mahmoud, Geomech. Eng. 11(5), 671-690 (2016)

48. A. Bouhadra, A. Tounsi, A.A. Bousahla, S. Benyoucef, S.R. Mahmoud, Struct. Eng. Mech. 66(1), 61-73 (2018) 
49. F. Bourada, K. Amara, A.A. Bousahla, A. Tounsi, S.R. Mahmoud, Struct. Eng. Mech. 68(6), 661-675 (2018)

50. A. Bouadi, A.A. Bousahla, M.A. Houari, H. Heireche, A. Tounsi, Adv. Nano. Res. 6(2), 147-162 (2018)

51. Y. Mokhtar, H. Heireche, A.A. Bousahla, M.A. Houari, A. Tounsi, S.R. Mahmoud, Smart Struct. Syst. 21(4), 397-405 (2018)

52. M. Yazid, H. Heireche, A. Tounsi, A.A. Bousahla, M.A. Houari, Smart Struct. Syst. 21(1), 15-25 (2018)

53. K. Bouafia, A. Kaci, M.A. Houari, A. Benzair, A. Tounsi, Smart Struct. Syst. 19(2), 115-126 (2017)

54. A. Zemri, M.A. Houari, A.A. Bousahla, A. Tounsi, Struct. Eng. Mech. Int. J. 54(4), 693-710 (2015)

55. M. Ahouel, M. A. Houari, E. A. Adda Bedia, A. Tounsi, Steel Compos. Struct. 20(5), 963-981 (2016).

56. H. Bellifa, K.H. Benrahou, A.A. Bousahla, A. Tounsi, S.R. Mahmoud, Struct. Eng. Mech. 62(6), 695-702 (2017)

57. B. Kadari, A. Bessain, A. Tounsi, H. Heireche, A.A. Bousahla, M.A. Houari, J. Nano Res. 55, 42-56 (2018)

58. B. Karami, M. Janghorban, A. Tounsi, Thin Wall. Struct. 129, 251-264 (2018)

59. B. Karami, M. Janghorban, A. Tounsi Steel. Compos Struct. 27(2), 201-216 (2018)

60. B. Bakhadda. M. B. Bouiadjra, F. Bourada, A. A. Bousahla, A Tounsi, Wind Struct. 27(5), 311-324 (2018).

61. F. Bounouara, K.H. Benrahou, I. Belkorissat, A. Tounsi, Steel Compos. Struct. 20(2), 227-249 (2016)

62. F.L. Chaht, A. Kaci, M.A. Houari, A. Tounsi, O.A. Beg, Steel. Compos. Struct. 18(2), 425-442 (2015)

63. X. Zhang, P. Agarwal, Z. Liu, H. Peng, Open Math. 13(1), 908-923 (2015)

64. P. Agarwal, S.S. Dragomir, M. Jleli, B. Samet Basel: Birkhäuser, (2018)

65. M. Ruzhansky, Y.J. Cho, P. Agarwal, I. Area, Springer, (2017)

66. P. Agarwal, I.H. Ibrahim, F.M. Yousry, 103, 1-15 (2019)

67. S. Rekhviashvili, A. Pskhu, P. Agarwal, S. Jain, Turkish Journal of Physics 43(3), 236-242 (2019)

68. Z. Hammouch, T. Mekkaoui, P. Agarwal, The European Physical Journal Plus 133(7), 248 (2018)

69. M. Ruzhansky, Y.J. Cho, P Agarwal (I Area, Springer Singapore, 2017)

70. P. Agarwal, A.A. El-Sayed, Phys A 500, 40-49 (2018)

71. P. Agarwal, A. Berdyshev, E. Karimov, RM 71(3-4), 1235-1257 (2017)

72. X. Zhang, P. Agarwal, Z. Liu, H. Peng, F. You, Y. Zhu, Advances in Difference Equations (1), 123, 2017

73. V.F. Morales-Delgado, J.F. Gómez-Aguilar, K.M. Saad, M.A. Khan, P. Agarwal, Phys A 523, 48-65 (2019)

74. U. Baltaeva, P. Agarwal, Mathematical Methods in the Applied Sciences 41(9), 3307-3315 (2018)

75. H. Zhou, L. Yang, P. Agarwal, Journal of Applied Mathematics and Computing 53(1-2), 51-76 (2017)

76. P. Agarwal, E. Karimov, M. Mamchuev, M. Ruzhansky, 707-718 (Birkhäuser, Cham, 2017)

77. K Saoudi, P Agarwal, P Kumam, A. Ghanmi, P. Thounthong, Advances in Difference Equations (1), 263, (2018)

78. J. Tariboon, S.K. Ntouyas and B. Sutthasin, Advances in Difference Equations (1), 220, (2019)

79. E.T. Thostenson, W.Z. Li, D.Z. Wang, Z.F. Ren, T.W. Chou, J. Appl. Phy. 91(9), 6034-6037 (2002)

80. H.S. Shen, Compos. Struct. 91(3), 375-384 (2009)

81. M. Kim, Y.B. Park, O.I. Okoli, C. Zhang, C. Compos. Sci. Tech. 69(3), 335-342 (2009)

82. C. Feng, S. Kitipornchai, J. Yang, Compos. Part B- Eng. 110, 132-140 (2017)

83. M. Rafiee, J. Yang, S. Kitipornchai, Compos. Struct. 96, 716-725 (2013)

84. J. Qiu, Y. Li, C. Chang, S. Atobe, H. Fukunaga, Y. Liu, H. Ning, L. Wu, J. Li, W. Yuan, T. Watanabe, C. Yan, Y. Zhang, N.-H. Alamusi, Nano. Res. Lett. 8, 1-8 (2013)

85. H.S. Shen, C.L. Zhang, Mater. Des. 31, 3403-3411 (2010)

86. K. Mayandi and P. Jeyaraj, J Mater Des Appl. 1-16 (2013).

87. H.S. Shen, D.Q. Yang, Appl. Math. Model. 39, 1480-1499 (2015)

88. S. Sahmani, M.M. Aghdam, Int J Mech Sci. 131, 95-106 (2017)

89. L.L. Ke, Y.S. Wang, J. Yang, S. Kitipornchai, Acta Mech. Sin. 30(4), 516-525 (2014)

90. M. Vinyas, Compos. Struct. 208, 600-617 (2019)

91. M. Vinyas, G. Nischith, M.A.R. Loja, F. Ebrahimi, N.D. Duc, Compos. Struct. 214, 132-142 (2019)

92. M. Vinyas, A.S. Sandeep, N.T. Trung, F. Ebrahimi, N.D. Duc, J. Intell. Mater. Syst. 30(6), 2478-2501 (2019) 